\title{
AVALIAÇÃO DO MÓDULO DE RESISTÊNCIA EM PEÇAS ESTRUTURAIS ROLIÇAS E EM CORPOS DE PROVA DE MADEIRA DE PINUS ELLIOTTII
}

\author{
André Luiz Zangiácomo ${ }^{1}$ \\ André Luis Christoforo ${ }^{2}$ \\ Francisco Antonio Rocco Lahr $^{3}$
}

\begin{abstract}
Resumo:O documento normativo brasileiro em vigor ABNT NBR 7190:1997 (Projeto de estruturas de madeira) especifica que os cálculos estruturais podem ser realizados a partir de resultados obtidos em ensaios realizados em corpos de prova de pequenas dimensões e isentos de defeitos, como da presença de nós, que podem ocorrer em peças estruturais e influenciar nos seus desempenhos. Assim, este trabalho avalia os valores de módulos de resistência (MOR) obtidos a partir de ensaios de compressão paralela às fibras realizados em corpos de prova isentos de defeitos e em peças estruturais roliças de madeira da espécie Pinus elliottii. Foram ensaiados quarenta e oito exemplares, sendo vinte e quatro de pequenas dimensões e vinte e quatro de dimensões estruturais. A análise de variância (ANOVA) revelou equivalência estatística entre os valores médios do MOR obtidos dos corpos de prova (16MPa) e das peças estruturais (15MPa). Os resultados indicam que os ensaios sobre os corpos de prova de dimensões reduzidas podem conduzir a cálculos estruturais satisfatórios, revelando neste estudo, para as quantidades de peças avaliadas assim como da espécie utilizada à boa precisão do modelo de cálculo proposto pela norma brasileira, entretanto, podendo apresentar resultados diferentes para outras peças de madeira da mesma espécie ou de espécies diferentes, justificando o ensaio em peças de dimensões estruturais.
\end{abstract}

Palavras-chave: madeira; compressão paralela; análise de variância.

\section{EVALUATION OF THE MODULUS OF RUPTURE IN STRUCTURAL ROUND TIMBER AND SMALL CLEAR SPECIMENS OF PINUS ELLIOTTII WOODEN SPECIES}

\begin{abstract}
The Brazilian standard ABNT NBR 7190:1997 (Design of Timber Structures) specifies that structural design can be done using data obtained from small clear specimens. In these conditions, natural characteristics, as knots, may affect the mechanical properties. The aim of this research is to evaluate the modulus of rupture (MOR) obtained in compression parallel to grain, carried out for small clear specimens and structural round timber of Pinus elliottii wood specie. Twenty four compression tests were carried out in small clear specimens and twenty four in structural round timber pieces. ANOVA analysis indicates that there is no statistical difference between the mean values obtained for the small clear specimens (16MPa) and structural pieces $(15 \mathrm{MPa})$. The results indicate that the samples may lead to satisfactory structural calculations in the case of compression parallel to grain, revealing this study, to number of samples evaluated as well as the species used, the good accuracy of calculation model proposed by Brazilian standard to obtain the modulus of rupture in compression parallel to grain, however, may provide different results for other pieces of the same or different species, justifying the tests in samples with structural dimensions.
\end{abstract}

Keywords:wood; compression parallel to grain; analysis of variance.

${ }^{1}$ Departamento de Engenharia, Universidade Federal de Lavras. Rua 13 de outubro, 130, centro, 37200-000, Lavras - MG. andrezangiacomo@deg.ufla.br

${ }^{2}$ Departamento de Engenharia Mecânica, Universidade Federal de São João del-Rei. Praça Frei Orlando, 170, Centro, 36307-352, São João del Rei - MG. alchristoforo@ yahoo.com.br,

${ }^{3}$ Departamento de Engenharia de Estruturas, Escola de Engenharia da Universidade de São Paulo. Rua Trabalhador Sãocarlense, 400, centro, 13566-590, São Carlos - SP, frocco@ @c.usp.br

ENGEVISTA, V. 16, n. 2, p.173-179, Junho 2014 


\section{INTRODUÇÃO}

O emprego de elementos estruturais roliços na construção civil corresponde a um dos usos mais eficientes de recursos florestais. As peças de seção transversal circular são facilmente obtidas, pois correspondem à configuração geométrica natural das árvores, requerendo um menor número de operações durante o seu processamento. Existem diversas possibilidades do uso dos elementos roliços, destacando-se entre elas as estruturas para pontes, as estruturas aplicadas às redes elétricas aéreas de distribuição (postes e torres), pilares, estacas de fundações, habitações, coberturas, silos, galpões de armazenamento ou de confinamento animal e estruturas de contenção. No dimensionamento estrutural, a norma brasileira ABNT NBR 7190:1997 (Projeto de Estruturas de Madeira) orienta que os elementos de seção circular podem ser considerados como de seção quadrada de área equivalente, especificando as peças a partir de seus diâmetros de base e de topo.

Para o correto emprego da madeira como material estrutural, com vistas à segurança, à funcionalidade, à durabilidade e à economia, faz-se necessário o conhecimento das suas propriedades físicas e mecânicas.

A obtenção do módulo de resistência da madeira é feita a partir de ensaios padronizados no Anexo B da norma ABNT NBR7190:1997, realizados em corpos de prova de pequenas dimensões. Essas peças são confeccionadas excluindo-se imperfeições naturalmente presentes nas árvores, como nós de crescimento de galhos e desvios de fibras. Tal procedimento pode influenciar nos resultados obtidos nos ensaios, uma vez que os chamados "defeitos" são descartados nesses pequenos exemplares.

Faz-se necessário, então, o desenvolvimento de trabalhos de pesquisa para que sejam determinadas, de modo confiável, as propriedades mecânicas de elementos estruturais roliços oriundos de florestas plantadas, como subsídios fundamentais para engenheiros projetistas, corroborando para a disseminação do emprego dessas peças em estruturas para os mais variados fins.

Assim, o objetivo desse estudo consistiu em comparar os valores de módulos de resistência à compressão paralela obtidos de peças estruturais roliças e de corpos de prova isentos de defeitos em madeiras da espécie Pinus elliottii.

Nesse sentido, relevantes para esse trabalho são os estudos que tratam de propriedades mecânicas da madeira, sendo importantes também as pesquisas sobre elementos roliços, com emprego estrutural.

Alguns países, procurando atender à demanda de madeira para diversos fins, dentre os quais os estruturais, cortam suas árvores cada vez mais precocemente, o que acarreta na diminuição dos diâmetros das peças disponíveis. Dessa forma, observa-se a realização de trabalhos em madeiras roliças de pequenos diâmetros, como o de Wolfe e Moseley (2000), que avaliaram o emprego de peças de pequenos diâmetros de espécies de madeira para fins estruturais. Neste, os pesquisadores realizaram ensaios de flexão estática e de compressão paralela às fibras para as espécies Douglas-fir (Pseudotsugamenziesii), Ponderosa Pine (Pinus ponderosa) e White fir (Abiesconcolor), concluindo que os módulos de elasticidade e resistência estimados por intermédio do ensaio de flexão estática consistem em importantes parâmetros de avaliação para classificação das peças.

Também em peças roliças foram feitos os estudos de Ross et al. (2001), em que toras de duas espécies de madeira, Red Pine (Pinus banksiana Lamb.) e Jack Pine (Pinus resinosa Ait.), com diâmetros à altura do peito variando de 12 a 31 centímetros, foram ensaiadas na flexão estática, sendo empregados em conjunto os testes de vibração transversal e ondas de tensão na direção longitudinal (stress wave). Os autores concluem nesse trabalho que tais peças podem ser avaliadas com sucesso pelas técnicas não destrutivas.

Ensaios de flexão estática em elementos roliços em sua forma original, cônica, e em elementos de seção circular constante são feitos por Larson et al. (2004). Os resultados desse trabalho 
revelaram que modificar a forma cônica original de uma peça roliça em uma peça com diâmetro constante aumenta os valores de resistência e rigidez na flexão, pois ocorre um acréscimo da porcentagem de madeira juvenil na seção transversal.

Green et al. (2006) avaliaram propriedades de resistência e rigidez em madeiras roliças. Para tanto foram selecionadas 110 árvores, sendo 42 da espécie Alpinefir (Abieslasiocarpa), 40 de Lodgepole pine (Pinus contorta), 23 de Engelmannspruce (Piceaengelmannii), 2 de Western white pine (Pinus monticola) e 1 de Grand fir (Abiesgrandis). As árvores foram processadas originando amostras com diâmetro de $228 \mathrm{~mm}$ e comprimento variando de 4,3 a 4,9 m. As amostras foram submetidas aos testes de vibração transversal, flexão estática a três pontos (ASTM D198:1994) e compressão paralela, obtendo assim as resistências e os módulos de elasticidade dinâmico e estático. Dentre outras, os autores concluem que os módulos de elasticidade dinâmicos apresentaram ser próximos dos estáticos pelas boas correlações encontradas.

Fernández-Golfín et al. (2007) realizaram classificação mecânica em madeiras roliças de Pinus nigra. Para tanto, foram utilizadas 445 peças de madeira roliça com diâmetros de 80, 100, 120 e 140 $\mathrm{mm}$ e comprimentos de $2 ; 2,5$ e $3 \mathrm{~m}$. As peças foram submetidas a ensaios mecânicos até o ponto de falha, como preconiza a norma europeia EN 1425, obtendo assim os valores da resistência à flexão e do módulo de elasticidade.

Green et al. (2008) avaliaram as propriedades mecânicas de resistência e rigidez na flexão e compressão paralela em peças de madeira roliça segundo abordagens de cálculo estática e dinâmica, utilizando 160 árvores da espécie Pseudotsugamenziesiie 218 da espécie Pinus ponderosa. As vigas possuíam aproximadamente $4 \mathrm{~m}$ de comprimento e diâmetro variando entre 100 a $200 \mathrm{~mm}$. Da abordagem estática, os módulos de elasticidade e de ruptura foram obtidos seguindo os padrões de cálculo da norma ASTM D 198:2006, sendo os módulos de elasticidade dinâmicos calculado pelo método de vibração transversal. Após análise dos resultados, foram observadas correlações de 0,41 a 0,66 entre os módulos de elasticidade estáticos e dinâmicos e entre as resistências mecânicas.

No Brasil, estudos têm sido desenvolvidos nos últimos anos, incrementando o nível de conhecimento sobre os temas aqui tratados.

Um cenário da utilização da madeira roliça de eucalipto na arquitetura brasileira, através do levantamento de alguns sistemas construtivos, suas vantagens e desvantagens, e de obras executadas em diversas regiões do país, são apresentados por Cunha (2004).

Santos e Ballarin (2002) compararam os resultados de módulos de elasticidade obtidos em ensaios de corpos de prova na flexão estática e de compressão paralela às fibras, a partir de árvores com cerca de 30 anos das espécies Corymbiacitriodora, Eucalyptussaligna e Eucalyptusgrandis. Os autores concluem nesse trabalho que o módulo de elasticidade à flexão foi inferior ao módulo de elasticidade à compressão paralela, apresentando relações entre ambos variando de 0,75 a 0,78 .

Um estudo realizado por Miná et al. (2004), que avaliaram a resistência e a rigidez de postes de madeira Corymbia citriodora, comparando os resultados com os obtidos em corpos de prova isentos de defeitos, apontam que os valores obtidos nos ensaios de flexão estática são maiores para as peças estruturais $(22175 \mathrm{MPa})$ do que para as de pequenas dimensões (20078MPa), e que na compressão paralela os módulos de resistência dos corpos de prova $(21705 \mathrm{MPa})$ são maiores que os estruturais (19990MPa).

Zangiácomo e Rocco Lahr (2008) estudaram a relação entre o comprimento (L) e diâmetro (d) em vigas roliças de madeira para o qual o efeito das forças cisalhantes se torna desprezível no cálculo dos deslocamentos, chegando às relações 12 e 15 para as madeiras Pinus elliottii e Pinus caribaea respectivamente.

Merecem ainda menções os trabalhos de Carreira et al. (2010), que avaliaram valores do módulo de elasticidade em toras de madeira com técnica de ensaio não destrutiva; e de Brito e Calil Jr. (2010), que propõem um manual para projetos $\mathrm{e}$ 
construção de estruturas utilizando peças roliças de madeira de reflorestamento. Para a determinação da rigidez em peças roliças, citam-se também as pesquisas de Christoforo et al. (2011a), Christoforo et al. (2011b) e Christoforo et al. (2012), sendo investigadas a influência da posição das peças roliças no ensaio de flexão para a obtenção do módulo de elasticidade longitudinal, a obtenção do módulo de elasticidade longitudinal por métodos numéricos de cálculo e a influência de medidas de pequenos deslocamentos na determinação do módulo de elasticidade longitudinal em peças roliças de madeira de dimensões estruturais.

Apesar dos mencionados estudos, percebe-se uma lacuna no que diz respeito à análise de valores de módulo de resistência na compressão paralela em elementos estruturais roliços da espécie Pinus elliotti, bem como a comparação com valores obtidos para as peças de pequenas dimensões recomendadas pela norma brasileira ABNT NBR7190:1997, razão deste trabalho.

\section{MATERIAL E MÉTODOS}

A escolha da espécie Pinus elliottii foi determinada por esta ser oriunda de floresta plantada e disponível na região de São Carlos, no Estado de São Paulo.

As árvores foram obtidas junto à Estação Ecológica de Itirapina, da Secretaria do Meio Ambiente do Estado de São Paulo, e alguns fatores foram relevantes na determinação do número de exemplares: o fato das mesmas terem sido solicitadas sob a forma de doação; o transporte, limitado pelas dimensões das peças e o custo de sua mobilização. Optouse, então, por adotar um número mínimo de exemplares referido em documentos normativos.

Nesse sentido, Hellmeister (1973) aponta em seu trabalho que o número mínimo de árvores estudadas por espécies deve ser igual a dez, devendo ser obtidas por amostragem ocasional, assim como feito com as árvores utilizadas no presente estudo. Já a norma técnica americana ASTM D5536-94 sugere um número mínimo de 5 elementos para a amostra cujas árvores apresentem diâmetros maiores que 30 centímetros, e 10 árvores para a amostras com diâmetros inferiores ao valor citado. Já a norma brasileira ABNT NBR 7190:1997 estabelece um número de 12 corpos de prova para a caracterização mínima da resistência de um lote de madeira serrada cujo volume não seja superior a 12 metros cúbicos.

Em campo observou-se que as árvores avaliadas apresentavam diâmetro à altura do peito (DAP) médios em torno de 30 centímetros e 15 metros de comprimento (descartando-se a copa da planta), e com posse destas informações foi calculado o volume de uma peça, aproximadamente um metro cúbico.

Como seriam realizados também ensaios estruturais, ou seja, cada árvore seria praticamente um corpo de prova, e com base na literatura citada, optou-se finalmente pelo número de 12 árvores a serem adquiridas.

Na Estação Ecológica de Itirapina, um técnico determinou de qual área poderiam ser cortados os exemplares, uma vez que na região existiam talhões plantados com diferentes espécies e diferentes idades. O Pinus elliottii estava plantado no talhão de número 41 , com as árvores tendo 25 anos de idade.

Determinada a região de interesse, as peças foram escolhidas aleatoriamente dentro do talhão, evitando-se selecionar as plantas que cresceram nas bordas da área, uma vez que essas árvores podem apresentar defeitos de crescimento, como madeira de reação, fibras torcidas e fustes curvos.

Cada exemplar de Pinus foi dividido em duas partes. Tal procedimento explicase pelo fato de que o comprimento original das peças era excessivo para os ensaios estruturais propostos, e para contribuir também para uma economia de transporte, uma vez que os novos 24 elementos de 7,5 metros puderam ser transportados todos de uma vez por um caminhão.

Feito o carregamento, as peças foram levadas ao Laboratório de Madeiras e de Estruturas de Madeira (LaMEM) do Departamento de Engenharia de Estruturas (SET) da Escola de Engenharia de São 
Carlos (EESC), Universidade de São Paulo (USP), onde os ensaios foram realizados.

Após o descarregamento e armazenamento das peças de Pinus, iniciouse a série de ensaios estruturais. Os elementos roliços avaliados possuem densidade aparente média de $0,55 \mathrm{~g} / \mathrm{cm}^{3}$, comprimento médio de $710 \mathrm{~cm}$ e fator de forma médio da seção de 0,86 .

Devido ao cronograma previsto, os testes foram conduzidos em peças com valores de teor de umidade acima do ponto de saturação das fibras, na chamada condição de madeira verde. Com isso, buscou-se uma padronização dos resultados, descartando-se a possibilidade da influência do teor de umidade na composição dos mesmos.

$\mathrm{Na}$ sequência, foram realizados os ensaios de compressão paralela às fibras nas peças estruturais. Corpos de prova de aproximadamente 40 centímetros de comprimento foram retirados das peças de 7,5 metros e ensaiados em três ciclos de carregamento. Para cada exemplar, um corpo de prova gêmeo foi ensaiado para a estimativa da resistência. Para as medições das deformações foram colocados relógios comparadores em posições diametralmente opostas, conforme apresentado na Figura 1, que ilustra o ensaio de um elemento estrutural roliço.

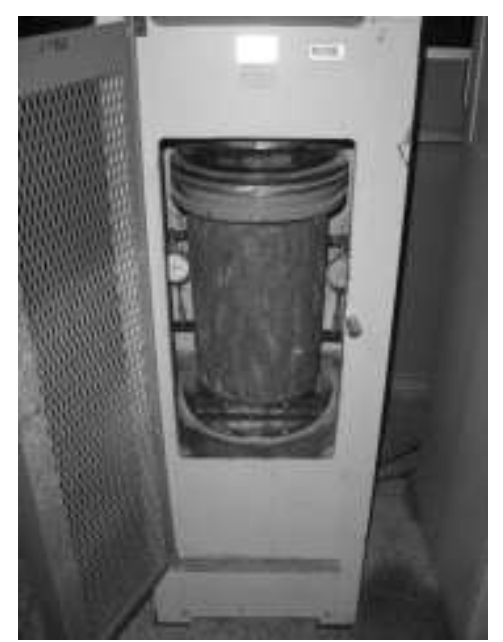

Figura 1: Ensaio de compressão paralela em elemento estrutural roliço.

Os ensaios descritos anteriormente foram realizados, na sequência, para corpos de prova de dimensões reduzidas obtidos dos elementos estruturais roliços. As dimensões das pequenas peças e as metodologias dos ensaios também seguiram as recomendações do documento normativo brasileiro ABNT NBR 7190:1997.

\section{RESULTADOS}

A Tabela 1 apresenta os valores médios $\left(\mathrm{X}_{\mathrm{m}}\right)$, desvios-padrão (DP), coeficientes de variação (CV) e valores máximos (Máx.) e mínimos (Mín.) dos módulos de resistência obtidos dos elementos estruturais roliços (Estrutural) e dos corpos de prova de pequenas dimensões e isentos de defeitos (Reduzido).

Tabela 1. Resultados dos módulos de resistência.

\begin{tabular}{ccc} 
Estatísticas & \multicolumn{2}{c}{ (MPa) } \\
& Est & PD \\
\hline $\mathrm{X}_{\mathrm{m}}$ & 15 & 16 \\
$\mathrm{DP}$ & 2,25 & 2,10 \\
$\mathrm{CV}(\%)$ & 15 & 13 \\
Mín. & 10,3 & 12,3 \\
Máx. & 19,7 & 19,3 \\
\hline
\end{tabular}

A Figura 2 ilustra os resultados dos testes de normalidade (Anderson-Darling) e de homogeneidade entre variâncias (Teste F), comprovando a normalidade nas distribuições dos valores de resistência e equivalência entre as variâncias pelos Pvalores encontrados serem ambos superiores aos níveis de significância dos testes $(\alpha=0,05)$, validando o modelo da ANOVA.

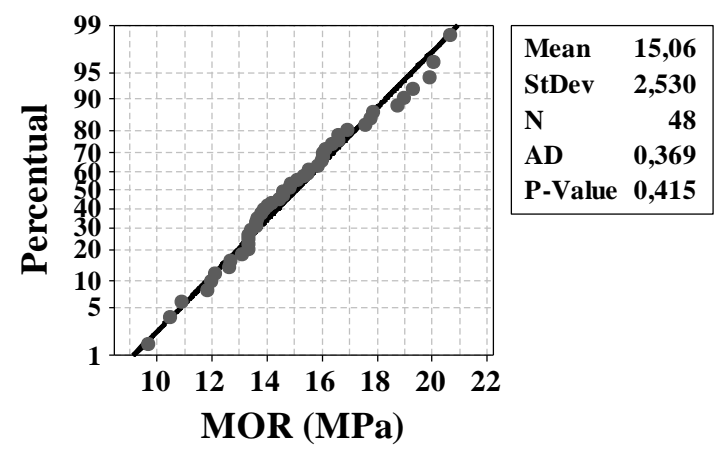

Figura 2. Teste de normalidade (a) 


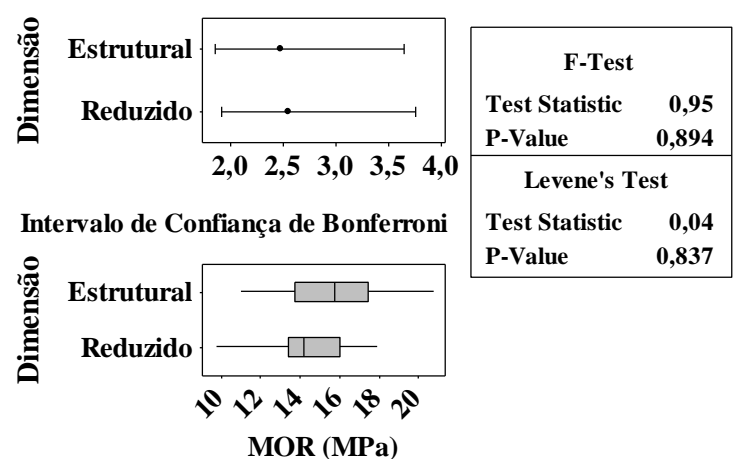

(b)

Figura 2. homogeneidade entre variâncias

(b) dos módulos de resistência.

A ANOVA do fator dimensão do corpo de prova (Estrutural; Reduzido) sobre os módulos de resistência apresentou P-valor igual a 0,103 (P-valor $>0,05)$, revelando serem as dimensões das amostras avaliadas não significativas (equivalentes). A Tabela 2 apresenta os resultados do teste de Tukey para os módulos de resistência à compressão paralela, sendo o gráfico de efeitos principais ilustrado na Figura 3.

Tabela 2. Resultados do teste de Tukey.

\begin{tabular}{ccccc} 
& \multicolumn{2}{c}{ MOR(MPa) } & \multicolumn{2}{c}{ Estatísticas } \\
\cline { 2 - 5 } & PD & Est & P-valor & F \\
\hline Agrup. & A & A & \multirow{2}{*}{2,77} & 0 \\
Média & 16 & 15 & 103 \\
\hline
\end{tabular}

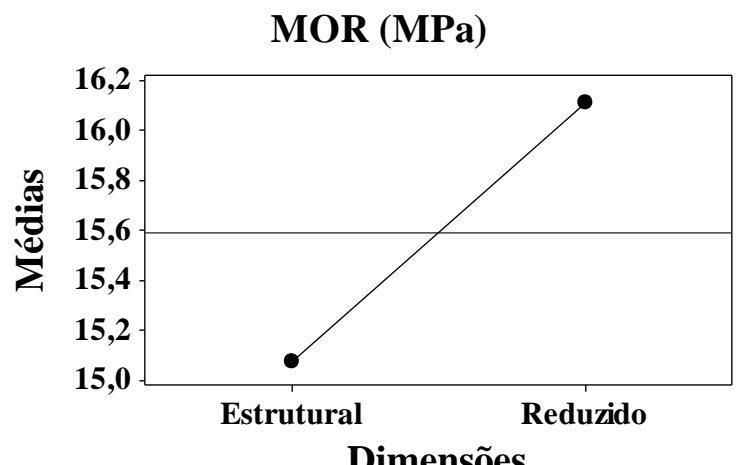

Figura 3. Gráfico de efeitos principais para o MOR.

Os resultados encontrados mostramse em consonância com os obtidos do trabalho de Miná et al. (2004), apresentando os corpos de prova com dimensões reduzidas os maiores valores médios da resistência na compressão paralela.

\section{CONCLUSÃO}

Os resultados obtidos do módulo de resistência na compressão paralela entre corpos de prova de dimensões reduzidas e os de dimensões estruturais para as madeiras Pinus elliottii avaliadas revelaram equivalência estatística, indicando neste caso a boa aproximação do modelo de cálculo da norma brasileira ABNT NBR 7190:1997. Entretanto, este resultado não pode ser estendido para outras espécies, podendo apresentar comportamentos diferentes dos encontrados nesta pesquisa, corroborando com a conveniência de se ter incluída, no texto proposto para revisão da norma ABNT NBR 7190:1997, a possibilidade de serem realizados ensaios de peças de dimensões estruturais para obtenção mais confiável das propriedades de resistência das madeiras avaliadas.

\section{REFERÊNCIAS}

AMERICAN SOCIETY FOR TESTING AND MATERIALS. ASTM D 198-05 Standard methods of static tests of lumber in structural sizes. Annual Book of Standards. West Conshohoken, PA, 2006.

AMERICAN SOCIETY FOR TESTING AND MATERIALS. ASTM D5536 Standard practice for sampling forest trees for determination of clear wood properties. Annual Book of Standards. Philadelphia, PA, 1994.

ASSOCIAÇÃO BRASILEIRA DE NORMAS TÉCNICAS. ABNT NBR 7190. Projeto de estruturas de madeira. Rio de Janeiro, 1997.

BRITO, L. D.; CALIL Jr., C. Manual de projeto e construção de estruturas com peças roliças de madeira de reflorestamento. Cadernos de Engenharia de Estruturas, São Carlos, v. 12, n. 56, p. 57-77, 2010.

CARREIRA, M. R.; SEGUNDINHO, P. G. A.; DIAS, A. A. Estimativa do módulo de elasticidade à flexão de toras de madeira por meio de vibração transversal livre. 
Madeira, Arquitetura e Engenharia, São Carlos, v.11, n.27, p.37-44, 2010.

CHRISTOFORO, A. L.; PANZERA, T. H.; BATISTA, F. B.; BORGES, P. H.; ROCCO, F. A. L.Avaliação numérica do módulo de elasticidade longitudinal de peças roliças estruturais de madeira Eucalyptus. Engenharia Agrícola, Jaboticabal, v.31, n.5, p.1007-1014, 2011 b.

CHRISTOFORO, A. L.; ROCCO, F. A. L.; MORALES, E. A. M.; ZANGIACOMO, A. L.; PANZERA, T. H.Influence of Displacements on Calculus of the Longitudinal Modulus of Elasticity of Pinus Caribaea Structural Round Timber Beams. International Journal of Agriculture and Forestry, v. 2, p.

157-160, 2012.

CHRISTOFORO, A. L.; PANZERA, T. H.; BATISTA, F. B.; BORGES, P. H.; ROCCO, F. A. L.; FRANCO, C. F.Influência da posição de peças roliças estruturais de madeira Eucalyptus no cálculo do módulo de elasticidade na flexão. Engenharia Agrícola, Jaboticabal, v.31, n.6, p.1219-1225, 2011a.

CUNHA, R. D. A. O eucalipto roliço na arquitetura brasileira. In: IX Encontro Brasileiro em Madeiras e em Estruturas de Madeira (EBRAMEM), Cuiabá, MT, 2004.

FERNÁNDEZ-GOLFÍN， J. I.; DÍEZBARRA, M. R.; HERMOSO, E.; MIER, R. Mechanical characterization of visually classified, small-diameter laricio pine round timber. Spanish Journal of Agricultural Research, v. 5, n. 3, p. 304-311, 2007

GREEN, D. W.; GORMAN, T. M.; EVANS, J. W.; MURPHY, J. F.; HATFIELD, C. A. Grading and properties of small-diameter Douglas-fir and ponderosa pine tapered logs. Forest Products Journal, v. 58, n. 11, p. 33-41, 2008.

GREEN, D. W.; GORMAN, T. M.; Evans, J. W.; Murphy, J. F. Mechanical Grading of Round Timber Beams. Journal of Materials in Civil Engineering, v. 18, n. 1, p. 1-10, 2006.

ENGEVISTA, V. 16, n. 2, p.173-179, Junho 2014
HELLMEISTER, J. C. Sobre a determinação das características físicas da madeira. Tese (Doutorado em Engenharia). Escola de Engenharia de São Carlos, Universidade de São Paulo, São Carlos, 161 p., 1973.

LARSON, D.; MIRTH, R.; WOLFE, R. Evaluation of small-diameter ponderosa pine $\operatorname{logs}$ in bending. Forest Products Journal, Madison, WI, v.54, p.52-58. 2004.

MINÁ, A. J. S.; DIAS, A. A.; CALIL Jr., C. Avaliação da rigidez e da resistência de postes de madeira para uso como estacas para fundações. In: IX Encontro Brasileiro em Madeiras e em Estruturas de Madeira (EBRAMEM), Cuiabá, MT, 2004.

ROSS, R. J.; ZERBE, J. I.; WANG, X.; GREEN, D. W.; PELLERIN, R. F. Comparison of several nondestructive evaluation techniques for assessing stiffness and MOE of small-diameter logs. Research Paper. Madison, WI: U. S. Department of Agriculture, Forest Service, Forest Products Laboratory. 2001.

SANTOS, E. S.; BALLARIN, A. W. Correlações entre os módulos de elasticidade à compressão paralela às fibras e à flexão para algumas espécies de eucalipto. In: VIII Encontro Brasileiro em Madeiras e em Estruturas de Madeira (EBRAMEM), Uberlândia, MG, 2002.

WOLFE, R.; MOSELEY, C. Smalldiameter $\log$ evaluation for value-added structural applications. Forest Products Journal. Madison, WI. v. 50. p. 48-58. 2000.

ZANGIÁCOMO. A. L, ROCCO LAHR, F. A. Avaliação do efeito do cisalhamento na flexão de elementos roliços da espécie Corymbia Citriodora. Anais In: XI Encontro Brasileiro em Madeiras e em Estruturas de Madeira, Londrina-PR, 2008. 